\title{
Two Years Remission of Type 2 Diabetes Mellitus after Bariatric Surgery
}

\author{
Nauman Wazir1, Muhammad Fahad Arshad4, John Finney2, Katie Kirk3 and Shivani Dewan1 \\ Department of Diabetes and Endocrine 1 / Nutrition and Dietics²/Surgery3, Doncaster Royal Infirmary and \\ Bassetlaw Teaching Hospitals, Doncaster DN2 5LT, UK \\ ${ }^{4}$ Department of Diabetes and Endocrine, Sheffield Teaching Hospitals, Sheffield, UK
}

\begin{abstract}
Objective: To determine the influence of bariatric surgery on remission of type 2 diabetes mellitus (T2DM) in obese patients along with improvements in other obesity-associated comorbidities.

Study Design: Observational study.

Place and Duration of Study: Doncaster and Bassetlaw NHS Trust, UK, from August 2010 to August 2018.

Methodology: All the cases of bariatric surgery in obese patients with T2DM who had completed 2 years of follow up were included in the study. Remission of T2DM was defined as glycated hemoglobin (HbA1C) $\leq 48 \mathrm{mmol} / \mathrm{mol}(\leq 6.5 \%)$ or fasting blood sugar of $\leq 7.0 \mathrm{mmol} / \mathrm{L}$, not on hypoglycemic agents 2 years after having bariatric surgery. Student's t-test was used to see any difference in baseline $\mathrm{HbA} 1 \mathrm{C}$, BMI, percentage of weight loss, and duration of diabetes between remitters and non-remitters.

Results: Two years follow-up data after bariatric surgery for remission of T2DM or otherwise was available for $(n=121)$ patients. Majority $(70.2 \%, n=85)$ were females and $(29.8 \%, n=36)$ were males. Mean age was $48.21 \pm 9.77$ years. Eightythree $(68.6 \%)$ patients achieved remission of T2DM at 2 years and $31.4 \%(n=38)$ did not. Remission of other comorbidities was $33.3 \%$ ( $n=53$ out of 159) for hypertension (HTN), 50.8\% ( $n=60$ out of 118 ) for dyslipidemia, $67.2 \%(n=43$ out of 64 ) for obstructive sleep apnea (OSA), $52.1 \%$ ( $n=37$ out of 71 ) for gastro esophageal reflux disease (GERD), $25.7 \%$ $(n=18$ out of 70$)$ for asthma, and $23.3 \%(n=24$ out of 103$)$ for depression.

Conclusion: Bariatric surgery effectively achieves remission of T2DM and other obesity associated comorbidities.
\end{abstract}

Key Words: Type 2 diabetes mellitus, Bariatric surgery, Roux-en-Y gastric bypass, Adjustable gastric band.

How to cite this article: Wazir N, Arshad MF, Finney F, Kirk K, Dewan S. Two years remission of type 2 diabetes mellitus after bariatric surgery. J Coll Physicians Surg Pak 2019; 29(10):967-71.

\section{INTRODUCTION}

Type 2 diabetes mellitus (T2DM) and obesity are global health problems which are on an increasing trend. T2DM has taken form of a global pandemic. Currently, 451 million people are estimated to have T2DM worldwide and by 2045 , it is predicted that around 693 million people, or one out of every 10 adults, will have diabetes. 1 The association of obesity with the development of T2DM as well as other comorbidities including dyslipidemia and hypertension has been clearly established. ${ }^{2}$ Although there is evidence for reduction of complications of type 2 diabetes with tight glycemic control, but at present all non-surgical therapies, including dietary and behaviour modification, exercise, oral hypoglycemic medication, and insulin, rarely causes remission of T2DM. ${ }^{3}$ On the other hand, bariatric surgery has been shown to have profound effects on glycemic control

Correspondence to: Dr. Nauman Wazir, Department of Diabetes and Endocrine, Doncaster Royal Infirmary and Bassetlaw Teaching Hospitals, Doncaster DN2 5LT, UK E-mail:nauman.wazir@yahoo.com

Received: November 06, 2018; Revised: March 04, 2019; Accepted: April 11, 2019 among people with type 2 diabetes mellitus, as suggested by numerous reports; some even suggest that surgery frequently produces a complete and sustained remission. 4

Bariatric surgeries can be of three principal types namely restrictive procedures including adjustable gastric band (AGB) and sleeve gastrectomy (SG); malabsorptive procedures namely, duodenojejunal bypass; and mixed procedures, which include Roux-en- $Y$ gastric bypass (RYGB), biliopancreatic diversion (BPD) and biliopancreatic diversion with duodenal switch (BPD-DS). The best results in terms of remission of T2DM have been reported with mixed techniques, such as RYGB, which is currently considered as a gold standard technique in obese diabetic patients. ${ }^{4,5}$ Restrictive techniques (AGB and $S G$ ) are alternative choice, which are on the rise due to simplicity of technique, considerably low rates of complications, and encouraging results which are sometimes comparable to RYGB.6,7

To know the impact of bariatric surgery on other obesityassociated comorbidities, while previous studies have mostly taken into account either one or two bariatric surgical procedures, the impact of all the three principal 
type of surgeries on remission of diabetes and other obesity-associated comorbidities was analyzed in this study.

The aim of this study was to find out short-term (2 years) remission of diabetes mellitus after bariatric surgery by analysing retrospective bariatric data of the hospital over a period of 8 years.

\section{METHODOLOGY}

It was a retrospective observational study. All the consecutive patients who had T2DM and underwent bariatric surgery over a period of eight years between August 2010 and August 2018 at Doncaster Royal Infirmary, and had completed their two years follow-up, were included in the study. Retrospective records of these patients were obtained from bariatric surgery database of the hospital. After being commissioned in April 2013 by NHS England, all the patients who had undergone bariatric surgery, fulfilled the NICE (National Institute of Clinical Excellence) guidelines for bariatric surgery. ${ }^{8}$ Before that point, only people with BMI of $\geq 50$ $\mathrm{Kg} / \mathrm{m}^{2}$ would be considered for bariatric surgery.

Inclusion criteria were age $\leq 68$ years or $\geq 18$ years and clinically diagnosed T2DM (According to WHO classification criteria of diabetes mellitus), 9,10 who underwent bariatric surgery procedures including RYBG, SG, and AGB occurring within the duration of the study period. Exclusion criteria were type 1 diabetes, gestational diabetes (with sole diabetes diagnosis), patients discharged from follow-up prior to two years after surgery and those having their surgical procedure reversed (e.g. removal of gastric band) in less than two years' time. Remission of T2DM was as per WHO classification criteria for diabetes mellitus, $, 9,10$ i.e. either fasting plasma glucose of less than $7 \mathrm{mmol} / \mathrm{L}$ or $\mathrm{HbA} 1 \mathrm{C}$ of less than $48 \mathrm{mmol} / \mathrm{mol}$, while not on any anti-diabetic medication. Remission of other comorbidities was considered for patients who had clinical/biochemical remission (as per NICE), or self-reported resolution of the symptoms of the respective conditions without taking medications.

Data was analysed using SPSS software version 23. Frequencies and percentage were used for qualitative variables like gender and status of remission of diabetes. Variables like duration of diabetes before surgery and percentage of weight loss after surgery were categorised into different strata. Mean, \pm SD $(95 \% \mathrm{Cl})$ were calculated for quantitative or numerical variables like age, BMI, HbA1C and duration of diabetes before surgery. Student's t-test was performed to detect difference in characteristics like baseline BMI, baseline $\mathrm{HbA} 1 \mathrm{C}$, duration of diabetes before surgery, and percentage of weight loss after surgery between the group of patients who achieved remission and the one of those who did not.
Table I: Baseline characteristics of study population $(n=121)$ who underwent bariatric surgery.

\begin{tabular}{l|c|c}
\hline Parameter & $\begin{array}{c}\text { Number(n) and } \\
\text { percentage }\end{array}$ & Mean \pm SD \\
\hline Age & & $48.21 \pm 9.77$ years \\
Gender & $36(29.8 \%)$ & \\
$\quad$ Males & $85(70.2 \%)$ & \\
Females & & $49.80 \pm 6.225 \mathrm{~kg} / \mathrm{m}^{2}$ \\
BMI & & $61.26 \pm 16.49 \mathrm{mmol} / \mathrm{mol}$ \\
Pre-Op HbA1C & & $5.98 \pm 4.53$ years \\
Duration of T2DM & & \\
BMI: Body Mass Index; Pre-Op HbA1C: Preoperative Glycated Heamoglobin; T2DM: Type 2 \\
Diabetes Mellitus.
\end{tabular}

\section{RESULTS}

A total of 598 patients underwent bariatric surgery from August 2010 to August 2018. Out of these patients, 191 had T2DM. Of these 191, 55 had not completed their 2-year follow-up, eight were lost to follow-up, two were discharged prior to completion of a 2-year follow-up, 2 had their gastric band removed before 2 years, and 3 died during the follow-up period. Therefore, we looked at data for 121 patients with T2DM, who underwent bariatric surgery and had a 2-year follow-up data until August 2018. Of these 121 patients, $14.87 \% \quad(n=18)$ underwent SG, $64.46 \%(n=78)$ underwent RYGB, 1.65\% $(n=2)$ underwent OAGB, and $19 \%(n=23)$ underwent AGB.

The baseline characteristic of patients $(n=121)$ are shown in Table I. Out of these 121 patients, $68.6(n=83)$ patients achieved remission of T2DM at 2 years after bariatric surgery and $31.4 \%(n=38)$ did not. Remission rates were higher in females $(71.8 \%, \mathrm{n}=61)$ as compared to males $(61.1 \%, n=22)$; and in those treated with oral hypoglycemic agents $(\mathrm{OHA})$ before surgery $(81.9 \%, n=68$ out of 83) as compared to those treated with insulin before surgery $(39.5 \%, n=15$ out of 38$)$.

Remission rates reduced as the duration of diabetes before surgery increased (Figure 1). Mean duration of diabetes in those who achieved remission after surgery was $4.38 \pm 3.87$ years, while it was $9.41 \pm 3.94$ years $(p=<0.0001)$ in those who did not achieve remission. On the other hand, remission rates increased with the percentage of weight loss after surgery (Figure 2). Mean percentage of weight loss 2 years after bariatric surgery was $29.55 \pm 10.44 \%$ in those who achieved remission after surgery; while it was $20.52 \pm 10.79 \%(p<0.001)$ in those who did not. While difference in percentage of weight loss seemed to be statistically significant, there was no statistical difference between mean baseline preoperative BMI of the subjects who achieved remission from diabetes after bariatric surgery and those who did not; mean BMI being $49.94 \pm 6.53 \mathrm{Kg} / \mathrm{m}^{2}$ and 49.90 $\pm 5.55 \mathrm{Kg} / \mathrm{m}^{2}$ ( $\left.p=0.72\right)$, respectively.

Preoperative glycemic control, i.e. the level of $\mathrm{HbA} 1 \mathrm{C}$, seemed to be a strong determinant of remission of diabetes postoperatively. Mean preoperative $\mathrm{HbA} 1 \mathrm{C}$ 


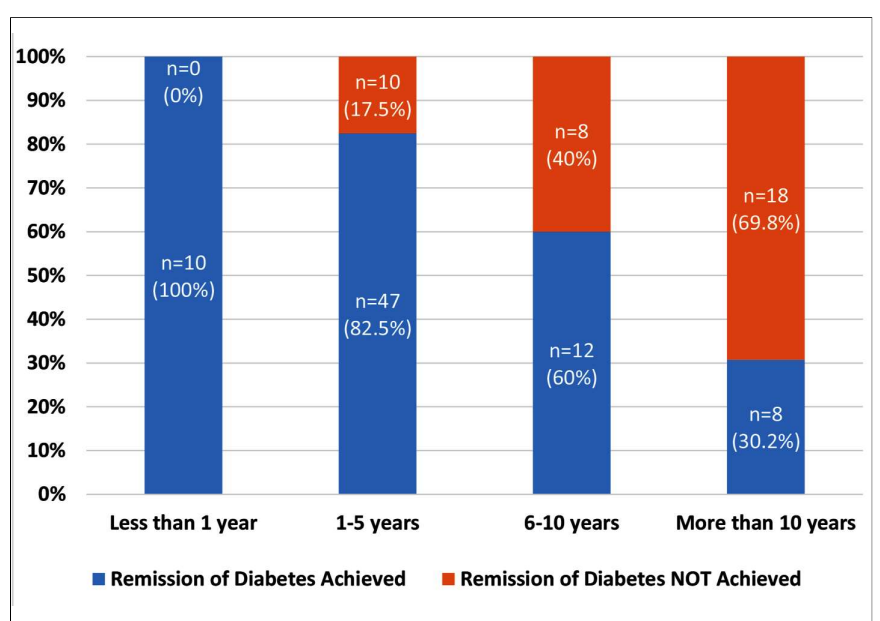

Figure 1: Remission of diabetes in different "duration of diabetes before bariatric surgery" categories.

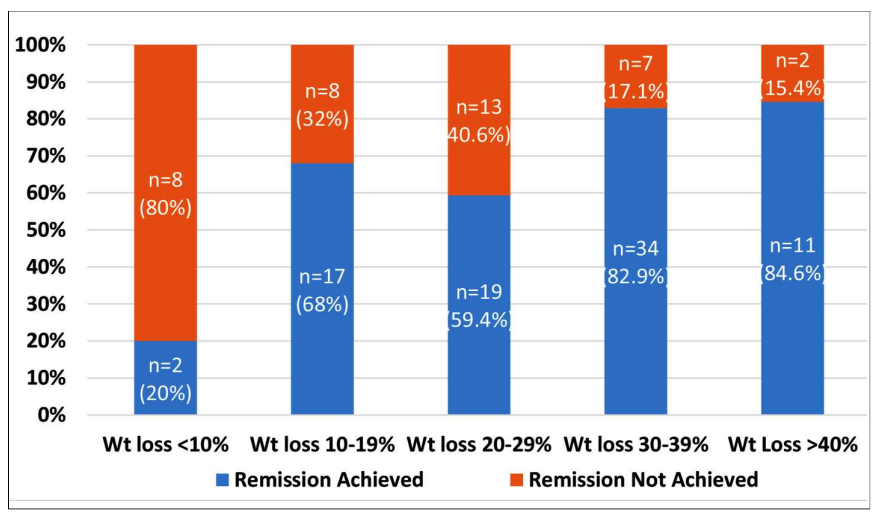

Figure 2: Remission of diabetes in various "percentage of weight loss after bariatric surgery" categories.

$\mathrm{Wt}=$ Weight.

was $56.97 \pm 14.82 \mathrm{mmol} / \mathrm{mol}$ in those who achieved remission 2 years after bariatric surgery, while it was $70.65 \pm 16.25 \mathrm{mmol} / \mathrm{mol}$ in those who did not $(p<0.001)$. Remission rates were highest for those who underwent RYGB: $80.8 \%(n=63)$, followed by SG: $55.6 \%(n=10)$, OAGB: $50 \%(n=1)$, and the least $39.1 \%(n=9)$ after gastric banding. Regarding remission of other comorbidities, 33.3\% $(n=53)$ patients with hypertension (HTN), 50.8\% ( $n=60)$ patients with dyslipidemia, 67.2\% $(n=43)$ patients with obstructive sleep apnea (OSA), $52.1 \%(n=37)$ patients with GERD, $25.7 \% \quad(n=18)$ patients with asthma, and $23.3 \%(n=24)$ with depression achieved remission of the respective conditions two years after bariatric surgery.

\section{DISCUSSION}

This study showed a considerable 2-year remission of T2DM $(68.6 \%)$ after bariatric surgery. Remission of T2DM in different types of bariatric surgery was in order of RYGB (80.8\%) >SG (55.6\%) >OAGB (50\%) >AGB $(39.1 \%)$. No meaningful discussion could be done about OAGB because of a very small sample, i.e. only two patients. However, rest of the results were in line with a meta-analysis which looked into results of bariatric surgery of 22,094 patients, showing that $76.8 \%$ patients had a complete resolution of diabetes. ${ }^{11}$ This metaanalysis showed an overall remission rate of diabetes of $83.75 \%, 71.6 \%$ and $47.9 \%$ with RYGB, SG and AGB, respectively.

Longitudinal assessment of bariatric surgery-2 (LABS-2), which was a large prospective cohort study, revealed that after a period of 3 years, $68.7 \%$ of RYGB and $30.2 \%$ of LAGB participants were in diabetes remission. ${ }^{12}$ Similarly, another systematic review showed remission rates of diabetes being higher with gastric bypass than gastric banding; $66.7 \%$ for gastric bypass and $28.6 \%$ for gastric band. 13 Lower rate of remission in the aforementioned study and the systematic review could be explained by the longer duration of follow-up in the study and that the systemic review took into account studies of a longer duration, i.e., at least 3 years. Diabetes remission rates fall with increasing duration after surgery. This fact is well elaborated in a retrospective cohort study, which showed an initial $70 \%$ remission of T2DM after RYGB within 5 years, but $35 \%$ of those achieving this remission redeveloped T2DM within the next 5 years. ${ }^{14}$ The present results are quite comparable to other studies looking into remission of T2DM at 2 years, 15,16 after gastric bypass and SG which found remission rates of $75 \%$ and $55.5 \%$, respectively almost similar to the present.

This study revealed that a higher proportion of females as compared to male patients underwent remission ( $71.8 \%$ and $61.1 \%$, respectively). This is in contrast to other studies which found female gender as a negative predictor of remission after bariatric surgery.17,18 This contradiction could be explained as a higher percentage of females $\{68.23 \% \quad(n=58)\}$ as compared to males $\{55.55 \%(n=20)\}$ undergoing RYGB, a procedure related to the highest remission rate of T2DM, in our study. This study showed that remission rate of T2DM after bariatric surgery was high in patients treated with oral hypoglycemic agents as compared to those who were treated with insulin before surgery. This result is quite consistent with the findings of a multisite study looking into long term remission and relapse of T2DM after gastric bypass. 14 This finding is probably related to a lower viable beta-cell mass and thus a lower endogenous insulin at the time of surgery in insulin-treated patients. ${ }^{19}$

This study found that the rate of remission of T2DM decreased with a longer duration of T2DM before surgery. Longer duration of diabetes prior to bariatric surgery has been consistently shown to be a negative predictive factor for remission of T2DM after bariatric surgery in numerous other studies. $11,16,20$

Difference in baseline mean BMI of the patients who achieved, and those who did not achieve remission of T2DM, was not statistically significant in this study. 
However, the difference in mean percentage of weight loss after bariatric surgery was statistically significant in these two groups. Remission rates of T2DM were found to be increasing as the percentage of weight loss after bariatric surgery increased. This finding is quite in agreement with the results of a multisite study by Arterburn et al., 14 who found that remission of diabetes is higher in patients who had a high percentage of weight loss after bariatric surgery, irrespective of the baseline BMI. Interestingly, same as the finding of our study, baseline BMI has not shown to be a predictor of diabetes remission at two years by an RCT conducted by Mingrone et al. ${ }^{16}$ More favourable outcomes, in term of remission of T2DM after bariatric surgery, are rather shown to be related to percentage of weight loss after surgery in various studies.6,20 In fact, Busetto et al. 19 inferred from the meta-analysis by Buchwald et al. ${ }^{4}$ that around $95 \%$ of the variability in the remission of diabetes, after various bariatric procedures, could be explained by the difference in weight loss as a result of these procedures.

Another important finding of this study was the statistically significant difference in level of glycemic control before surgery (in terms of $\mathrm{HbA1C}$ ), in patients who achieved remission of T2DM after bariatric surgery and those who did not. This is consistent with findings of earlier studies. ${ }^{6} \mathrm{HbA} 1 \mathrm{C}$, along with other factors namely, age, duration of diabetes, and use of other anti-diabetic agents, has been incorporated into a prognostic score (DiaRem) to predict the probability of diabetes remission by Still et al.21

Besides T2DM, this study showed that bariatric surgery leads to remission of other comorbidities, e.g. HTN, dyslipidemia OSA, GERD, depression and even asthma in significant proportion of patients. The SOS study has shown that the rates of remission of T2DM, HTN and dyslipidemia were significantly higher than control group 10 years after bariatric surgery. ${ }^{22}$ Likewise, beneficial impacts of bariatric surgery on OSA, GERD and depression have also been previously reported. ${ }^{23-25}$

\section{CONCLUSION}

Bariatric surgery leads to a considerable remission of T2DM as well as other obesity-related comorbidities. Female gender, shorter duration of diabetes before surgery, greater percentage of weight loss after surgery, treatment with OHA rather than insulin before surgery, and a lower baseline $\mathrm{HbA} 1 \mathrm{C}$ are associated with higher rates of remission of T2DM post-bariatric surgery.

\section{ETHICAL APPROVAL:}

Ethical approvals were obtained from "Health Research Authority (HRA) UK Research Ethics Committee (REC)" and "Doncaster Clinical Research, Doncaster and Bassetlaw Teaching Hospitals NHS Trust" prior to initiation of the research work.

\section{PATIENTS' CONSENT:}

Informed consents from patients are obtained to publish the data concerning this case as per Health Research Authority (HRA) UK Research Ethics Committee (REC) rules.

\section{CONFLICT OF INTEREST:}

Authors declared no conflict of interest.

\section{AUTHORS' CONTRIBUTION:}

NW: Main concept and design of the study, interpretation and analysis of data.

MFA: Drafting of manuscript.

JF: Acquisition of data.

KK: Acquisition of data.

SD: Critical revision of manuscript.

\section{REFERENCES}

1. IDF Atlas $8^{\text {th }}$ Edition, 2017. Available online: http://www. diabetesatlas.org.

2. Bastien M, Poirier P, Lemieux I, Després JP. Overview of epidemiology and contribution of obesity to cardiovascular disease. Prog Cardiovasc Dis 2015; 56:369-81.

3. Gregg EW, Chen H, Wagenknecht LE, Clark JM, Delahanty LM, Bantle $\mathrm{J}$, et al. Association of an intensive lifestyle intervention with remission of type 2 diabetes. JAMA 2012; 308:2489-96.

4. Buchwald $H$, Estok R, Fahrbach $K$, Banel D, Jensen MD, Pories WJ, et al. Weight and type 2 diabetes after bariatric surgery: Systematic review and meta-analysis. Am J Med 2009; 122:248-56.

5. Li P, Fu P, Chen J, Wang LH, Wang DR. Laparoscopic Roux-en-Y gastric bypass vs. laparoscopic sleeve gastrectomy for morbid obesity and diabetes mellitus: A meta-analysis of sixteen recent studies. Hepatogastroenterology 2013; 60:132-7.

6. Dixon JB, O'Brien PE, Playfair J, Chapman L, Schachter LM, Skinner $S$, et al. Adjustable gastric banding and conventional therapy for type 2 diabetes: A randomized controlled trial. JAMA 2008; 299:316-23.

7. Vidal P, Ramón JM, Goday A, Benaiges D, Trillo L, Parri A, et al. Laparoscopic gastric bypass versus laparoscopic sleeve gastrectomy as a definitive surgical procedure for morbid obesity. Mid-term results. Obes Surg 2013; 23:292-9.

8. Obesity: Identification, assessment and management (NICE guideline CG189), recommendation 1.10.1. https://www.nice.org.uk/ guidance/cg189.

9. World Health Organization. Definition and diagnosis of diabetes mellitus and intermediate hyperglycemia. Geneva: World Health Organization; 2006. http://www.who.int.

10. Report of a World Health Organization Consultation. Use of glycated haemoglobin $(\mathrm{HbA} 1 \mathrm{c})$ in the diagnosis of diabetes mellitus. Diabetes Res Clin Pract 2011; 93:299-309.

11. Buchwald $H$, Avidor $Y$, Braunwald $E$, Jensen MD, Pories $W$, Fahrbach $\mathrm{K}$, et al. Bariatric surgery: A systematic review and meta-analysis. JAMA 2004; 292:1724-37.

12. Purnell JQ, Selzer F, Wahed AS, Pender J, Pories W, Pomp A, et al. Type 2 diabetes remission rates after laparoscopic gastric bypass and gastric banding: Results of the longitudinal assessment of bariatric surgery study. Diabetes Care 2016; 39:1101-7. 
13. Puzziferri N, Roshek TB, Mayo HG, Gallagher R, Belle SH, Livingston $\mathrm{EH}$. Long-term follow-up after bariatric surgery: A systematic review. JAMA 2014; 312:934-42.

14. Arterburn DE, Bogart A, Sherwood NE, Sidney S, Coleman KJ, Haneuse $\mathrm{S}$, et al. A multisite study of long-term remission and relapse of type 2 diabetes mellitus following gastric bypass. Obes Surg 2013; 23:93-102.

15. Romero Lluch AR, Martínez-Ortega AJ, Socas-Macías M, Jiménez-Varo I, Pereira-Cunill JL, Serrano-Aguayo $\mathrm{P}$, et al. Resolution of type 2 diabetes and prediabetes following laparoscopic sleeve gastrectomy: Medium term results. Nutr Hosp 2014; 31:642-8.

16. Mingrone G, Panunzi S, De Gaetano A, Guidone C, laconelli A, Leccesi $\mathrm{L}$, et al. Bariatric surgery versus conventional medical therapy for type 2 diabetes. N Engl J Med 2012; 366:1577-85.

17. Dicker D, Yahalom R, Comaneshter DS, Vinker S. Long-term outcomes of three types of bariatric surgery on obesity and type 2 diabetes control and remission. Obes Surg 2016; 26: 1814-20.

18. Ramos-Levi A, Sanchez-Pernaute A, Matia P, Cabrerizo L, Barabash A, Hernandez C, et al. Diagnosis of diabetes remission after bariatic surgery may be jeopardized by remission criteria and previous hypoglycemic treatment. Obes Surg 2013; 23:1520-6.
19. Busetto L, Sbraccia P, Frittitta L, Pontiroli AE. The growing role of bariatric surgery in the management of type 2 diabetes: Evidences and open questions. Obes Surg 2011; 21:1451-7.

20. Cummings DE. Endocrine mechanisms mediating remission of diabetes after gastric bypass surgery. Int J Obes (Lond) 2009; 33(Suppl 1):S33-40.

21. Sjöström L, Lindroos AK, Peltonen M, Torgerson J, Bouchard C, Carlsson $\mathrm{B}$, et al. Lifestyle, diabetes, and cardiovascular risk factors 10 years after bariatric surgery. N Engl J Med 2004; 351:2683-93.

22. Still CD, Wood GC, Benotti P, Petrick AT, Gabrielsen J, Strodel WE, et al. Preoperative prediction of type 2 diabetes remission after RouxenY gastric bypass surgery: A retrospective cohort study. Lancet Diabetes Endocrinol 2014; 2:38-45.

23. Nagendran M, Carlin AM, Bacal D, Genaw JA, Hawasli AA, Birkmeyer NJ, et al. Self-reported remission of obstructive sleep apnea following bariatric surgery: Cohort study. Surg Obes Relat Dis 2015; 11:697-703.

24. El-Hadi M, Birch DW, Gill RS, Karmali S. The effect of bariatric surgery on gastroesophageal reflux disease. Can J Surg 2014; 57:139-44.

25. Sierzantowicz R, Lewko J, Hady HR, Kirpsza B, Trochimowicz L, Dadan J. Effect of BMI on quality of life and depression levels after bariatric surgery. Adv Clin Exp Med 2017; 26:491-6. 